\title{
Kardiovaskuläre Vorsorge im Breitensport, ambitionierten Freizeitsport und Wettkampfsport über das 35. Lebensjahr hinaus
}

\author{
Cardiovascular screening for recreational, leisure, vigorous and \\ competitive sport activities over 35 years
}

Autoren

Institut

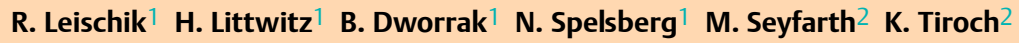

1 Universität Witten/Herdecke, Faculty of Health, School of Medicine, Hagen, Germany

2 Universität Witten/Herdecke, Faculty of Health, School of Medicine, Kardiologische Klinik Helios Wuppertal, Germany
Kardiologie, Sportmedizin

Schlüsselwörter

Marathon

Triathlon

Vorsorge Breitensport

Wettkampfsport

körperliche Aktivität

Keywords

marathon

triathlon

pre-participation screening

$\checkmark$ middle-aged athletes

physical activity

\section{Einführung \\ $\nabla$}

Die Inzidenz des plötzlichen Herztodes (PHT) in Verbindung mit Sport nimmt mit dem Alter zu [41]. PHT durch körperliche Anstrengung ist schon seit dem Altertum bekannt: Ein Soldat namens Pheidippides soll im Alter von etwa 35 Jahren nach der Schlacht bei Marathon nach Athen gelaufen und gestorben sein als er die Siegesnachricht überbrachte. Ob er Soldat war oder ein Bote, der vorher $240 \mathrm{~km}$ von Athen nach Sparta lief, dann über Marathon nach Athen zurück kam, und was letztendlich Wahrheit, was Dichtung ist, bleibt unklar [22]. Fakt ist, dass dieser Todesfall als der erste sportbezogene Tod in die Geschichte eingegangen ist.

Die Wahrscheinlichkeit des PHT in Verbindung mit Sport liegt innerhalb der Durchschnittsbevölkerung (Frankreich) bei ca. 4,6/1 Mio. Einwohner/Jahr [43]. Der PHT allgemein hat in der industrialisierten Welt eine Inzidenz von 0,36 bis 1,28/1000/Jahr [50].
Körperliche Inaktivität wird bei verschiedenen Erkrankungen als Mitauslöser angesehen, zum Beispiel beim Diabetes mellitus in 27\% der Fälle und bei der koronaren Herzerkrankung in ca. $30 \%$ der Fälle [64]. Körperliche Inaktivität ist in den USA für jährlich mindestens 150000 aller Todesfälle verantwortlich [12]. Körperliche Aktivität hingegen senkt das Gewicht, wirkt sich günstig auf den Diabetes mellitus aus [53] und senkt die kardiale Mortalität [57].

In der Literatur wird eine willkürliche (fließende) Grenze zwischen jungen und alten Athleten bei 35 Jahren gezogen $[8,43,45]$. Obwohl viele positive Einflüsse auf die Lebenserwartung bekannt sind, führen Berichte über Todesfälle bei Freizeitsportlern [2] und Artikel über das gesteigerte Risiko eines PHT durch Sport [43] zu einer gewissen Verunsicherung. Die mit Marathon [31] und Triathlon [27] assoziierten Todesfälle und die Todesfälle bei jungen Athleten ( Tab.1) verunsichern die ambitionierten Sportler im Amateur- und Profibereich.

Tab.1 Ursachen der kardial bedingten plötzlichen Todesfälle bei jungen Sportlern. Angaben in Prozent.

eingereicht 17.03.2014 akzeptiert 18.06 .2014

\section{Bibliografie}

DOI 10.1055/s-0034-1387346 Dtsch Med Wochenschr 2014; 139: 2188-2194 - (c) Georg Thieme Verlag KG · Stuttgart . New York · ISSN 0012-0472

Korrespondenz PD Dr. med. Roman Leischik Lehrauftrag Prävention, Gesundheitsförderung und Sportmedizin Universität Witten-Herdecke Elberfelder Str. 1 58095 Hagen

Tel. 02331/182500

Fax 02331/182570

eMail info@dr-leischik.de

\begin{tabular}{|c|c|c|c|c|}
\hline \multirow[t]{3}{*}{ Mögliche Ursachen für plötzlichen Herztod } & \multicolumn{4}{|l|}{ Studie } \\
\hline & Maron 2009 & Corrado 2003 & Solberg 2010 & Marijon 2011 \\
\hline & [67]; $n=1049$ & [8]; $n=55$ & {$[68] ; n=22$} & [43]; $n=50$ \\
\hline hypertrophische Kardiomyopathie & 36 & 1,8 & 4,3 & 10 \\
\hline koronare Herzerkrankung & 3 & 18 & 48 & 6 \\
\hline ARVK & 4 & 22 & - & 4 \\
\hline koronare Anomalie & 24 & 11 & 4,3 & \\
\hline mögliche hypertrophe Kardiomyopathie & 11,3 & - & - & 4 \\
\hline RIVA-Muskelbrücke & 2,3 & 3,6 & - & 2 \\
\hline Myokarditis & 5,4 & 9 & 22 & 4 \\
\hline Aortenruptur/Herzruptur & 2,2 & 1,8 & 4,3 & 2 \\
\hline Aortenstenose/angeb. Vitium & 2,6 & - & 4,3 & 6 \\
\hline Mitralklappenprolaps & 2,4 & 7,3 & - & 2 \\
\hline dilatative Kardiomyopathie & 2 & 1,8 & - & 4 \\
\hline unklar & - & 1,8 & - & 36 \\
\hline Überleitung/QT/WPW & 4,1 & 1,8 & 8,7 & 12 \\
\hline
\end{tabular}




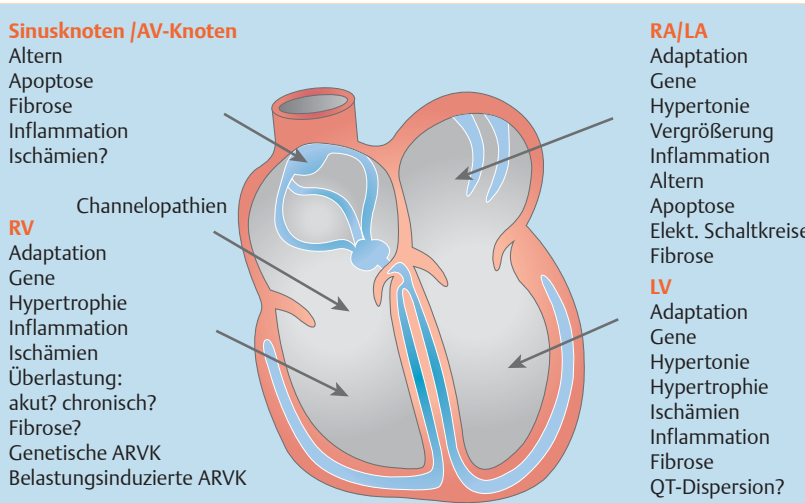

Abb.1 Mögliche Einflussfaktoren auf die kardialen Strukturen bei der Problematik Sport und kardiale Schädigung.

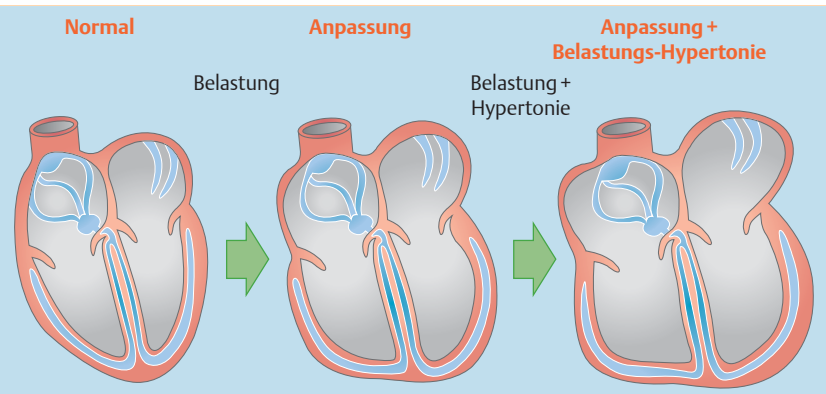

Abb.2 Negativer Einfluss der Belastungshypertonie beim Ausdauerathleten auf die kardialen Strukturen. Belastungshypertonie führt zur zusätzlichen Vergrößerung der Herzkammern und zusätzlichen Hypertrophie.

Berichte über eine potenzielle kardiale Schädigung durch Sport $[34,47]$ und diskutierte Faktoren, die zur Herzschädigung durch Sport führen können ( Abb.1), verlangen nach einem adäquaten Screening der an Sport Interessierten und Aktiven.

\section{Das Sportlerherz und seine möglichen Komplikationen im Alter}

Die kardiale Adaptation an die Belastung ist ein bekanntes Phänomen [24] und wurde vom finnischen Arzt Henschen Ende des 19. Jahrhunderts erstmals erwähnt [29]. Bei der physiologischen Veränderung des Sportlerherzens handelt es sich um eine harmonische Größenzunahme und Myokardhypertrophie eines gesunden Herzens, welche ausschließlich durch körperliche Aktivität bedingt sind [13].

Diese Vorstellung ist sehr vereinfacht und beruht in erster Linie auf der Herzmuskelhypertrophie. Problematisch ist, dass die Herzmuskelhypertrophie (und das „Sportlerherz“) kaum „nur“ durch körperliche Aktivität entsteht. Bei der sportbedingten Herzhypertrophie spielen weitere Faktoren wie Genetik [26], Art des ausgeübten Sports und belastungsinduzierter Hochdruck [40] eine wichtige Rolle ( Abb.2).

Bei einer 5-fach erhöhten Sterblichkeit der jungen Wettkampfathleten [43] stellen die myokardiale Hypertrophie und eine mögliche QT-Dispersion ein potenzielles Risiko dar [28]. 40\% der Athleten weisen abnorme EKG-Veränderungen auf [44]. Dabei werden hauptsächlich verlängerte QRS-Komplexe, diverse T-Wellen-Ver-

\begin{tabular}{|c|c|c|}
\hline $\begin{array}{l}\text { Sinusbradykardie } \geq 35 / \text { min } \\
\text { AV-Block I. Grades } \\
\text { inkompletter RSB } \\
\text { frühe Repolarisation } \\
\text { alleinige LV-Hypertrophie } \\
\text { Zeichen } \\
\text { T-Negativierung V1 - V4 } \\
\text { bei } \leq 14 \text {-Jährigen }\end{array}$ & $\begin{array}{l}\text { Abweichung Lagetyp nach } \\
\text { rechts/links } \\
\text { tiefe T-Negativierung bis V4 } \\
\text { bei Männern afrokaribischer } \\
\text { Abstammung } \\
\text { tiefe T-Negativierung bis V3 } \\
\text { bei Frauen afrokaribischer } \\
\text { Abstammung } \\
\text { Vergrößerung } \\
\text { linker Vorhof } \\
\text { rechter Vorhof } \\
\text { Sinusbradykardie <35/min }\end{array}$ & $\begin{array}{l}\text { ST-Strecken-Senkung } \\
\text { pathologische Q-Zacke } \\
\text { QTc } \geq 470 \text { ms ơ } 1 \geq 480 \text { ms } \text { + } \\
\text { atriale/ventrikuläre } \\
\text { Arrhythmien } \\
\text { T-Negativierung >V1 bei } \\
\text { Kaukasiern } \\
\text { T-Negativierung >V4 bei } \\
\text { afrokaribischer Abstammung } \\
\text { Hinweise auf Brugada- } \\
\text { Syndrom } \\
\text { RV-Hypertrophie } \\
\text { kompletter RSB/LSB } \\
\text { ventrikuläre Extrasystolen } \\
\text { (VES) } \\
\text { EKG-Veränderungen im } \\
\text { Verlauf }\end{array}$ \\
\hline
\end{tabular}

Abb.3 12-Kanal-Ruhe-EKG-Ampel. Grünes Licht: unbedenklich; gelbes Licht: grenzwertig positiver Befund, abnorme/pathologische Befunde möglich; rotes Licht: sportunabhängige Veränderungen, abklärungsbedürftig. LV = linker Ventrikel, RV = rechter Ventrikel, RSB = Rechtsschenkelblock, LSB = Linksschenkelblock, VES = ventrikuläre Extrasystolen (basierend auf $[51,9,22]$ und eigenen Erfahrungen).

änderungen (Negativierungen) und tiefe Q-Wellen bis hin zu „bizarren“ EKGs berichtet [48]. Die Häufigkeit der EKG-Veränderungen ist abhängig von der ethnischen Zugehörigkeit [52], der Art/Intensität des Trainings und der Sportart. EKG-Veränderungen sollen entsprechend den „Seattle-Kriterien“ [15] oder den europäischen Empfehlungen $[9,23,52]$ analysiert werden. QT-Längen $\geq$ 470 ms brauchen immer eine individuelle Betrachtungsweise und mindestens anamnestische familiäre oder sogar genetische Untersuchungen. Veränderungen im Verlauf müssen immer genau nachverfolgt werden. Die EKG-Veränderungen sind bezüglich ihrer Bedeutung in Abb.3 als Ampelschema beschrieben.

Die Haupt-Komplikationen des „Sportlerherzens“ im Alter sind Vorhofflimmern [7], supraventrikuläre Bradyarrhythmien [1] und nodale Erkrankungen [3]. Es gibt zahlreiche Berichte über Fibrosierungen des linken Ventrikels bei älteren Ausdauersportlern [60]; die Ursachen hierfür werden noch diskutiert [63]. Veränderungen an den Herzklappen bei Sportlern (beschleunigte Zunahme des Stenosegrades der Aortenklappe im Alter), Ektasie der Aorta und Vergrößerung der Vorhöfe stellen nur einige Veränderungen dar, die echokardiographisch leicht feststellbar sind. Funktionelle Veränderungen sind entweder mittels DopplerTechnik oder Strain-Technologie im Verlauf gut zu untersuchen.

\section{kurzgefasst}

Körperliche Inaktivität verursacht bis zu 30\% der Fälle Diabetes mellitus oder koronare Herzerkrankung. Sport kann bei einer Veranlagung zu einer pathologischen Form des Sportlerherzens führen und im Alter für Herzrhythmusstörungen/Vorhofflimmern verantwortlich sein. Daneben können Inflammationen und Belastungshypertonie Ursachen darstellen. Das Ruhe-EKG kann die ersten Hinweise auf eine evtl. pathologische Situation liefern. 


\section{Plötzlicher Herztod im Sport und die Lebenserwartung der Sportler \\ $\nabla$}

Plötzlicher Herztod im Sport

Die Häufigkeit des PHT beim Sport steigt mit dem Alter. In der Gruppe der 15- bis 34-Jährigen liegt die Mortalität bei 2,96/1 Mio./Jahr (95\%-Konfidenzintervall [KI] 2,50-3,42), bei den 35bis 54-Jährigen bei 6,63/1 Mio./Jahr (95\%-KI 5,94-7,31) und bei den 55- bis 75-Jährigen bei 7,51/1 Mio./Jahr (95\%-KI 6,60-8,43) [41]. Die älteren Sportler sind ca. doppelt so häufig betroffen wie die jüngeren und Männer ca. 15- bis 20-mal häufiger als Frauen [41]. Bei Athleten über 35 Jahren ist der sportbezogene plötzliche Tod meistens kardial bedingt [61].

Der PHT bei Volksläufen tritt insgesamt selten auf (1/129 000 Laufstunden, 95\%-KI 1/62 500-1/263 000) [46]. Marijon et al. beschreiben das allgemeine Risiko des PHT in Verbindung mit Sport je nach Region mit 5,4 bis 16,7/1 Mio./Jahr [43]. Das mittlere Alter der Betroffenen lag bei $46 \pm 15$ (11-75) Jahren, 92\% starben direkt beim Sport, nur $12,7 \%$ hatten vorher Beschwerden und 86,5\% waren regulär im Training. Bei der allgemeinen Bevölkerung liegt das Risiko bei Männern im Bereich von 9,2/1 Mio./Jahr, bei Frauen bei 0,4/1 Mio./Jahr.

Bei Langdistanzläufen in den USA (Halbmarathon/Marathon) starben zwischen 2000 und 2010 von 10,9 Mio. Läufern 59 Personen (Inzidenzrate 0,54/100 000 Teilnehmer [95\%-KI 0,41$0,70]$ ). Die Betroffenen waren im Durchschnitt $42 \pm 13$ Jahre alt und überwiegend männlich (51 vs. 8 Frauen) [31]. Die Inzidenzrate für den PHT lag beim Marathon bei 1,01/100 000 (95\%-KI $0,72-1,38$ ) und beim Halbmarathon bei $0,27 / 100000$ (95\%-KI, $0,17-0,43)$. Männer waren häufiger betroffen als Frauen (0,9/100 000; 95\%-KI 0,67-1,18 vs. 0,16/100 000; 95\%-KI 0,070,31 ) [31]. Die Autoren berichten in dieser Studie, dass nicht unbedingt eine Plaqueruptur, sondern die Dysbalance zwischen dem myokardialen Sauerstoffangebot und -verbrauch die Ursache ist [31].

Bei jungen Sportlern trat der PHT mit 9,8/1 Mio./Jahr, bei jungen Nichtsportlern mit 2,2/1 Mio./Jahr auf. Junge Wettkampfsportler haben folglich ein 5-fach höheres Risiko als Nicht-Wettkampfsportler [43]. Bei jungen Athleten in den USA treten pro Jahr 50-75 Todesfälle auf [45].

Diese Tatsachen unterstützen die Ansicht, dass alle Wettkampfsportler unabhängig vom Alter genauer betrachtet werden sollten, um die Risikopersonen zu identifizieren. Eine „negative Triggerung“ der bestehenden Disposition kann zum PHT führen. Möglicherweise spielt hier die individuelle Veranlagung, eine pathologische Hypertrophie, das persönliche Risikoprofil, eine Elektrolyt-Verschiebung oder Inflammation eine Rolle (O Abb.1).

\section{Lebenserwartung der Sportler}

Die Arbeit von Marijon at al. [42] berichtet über eine um 41\% niedrigere Gesamtmortalität von Profi-Radfahrern im Vergleich zur allgemeinen Bevölkerung. Auch eine aktuelle Meta-Analyse berichtet über eine geringere (vor allem kardiovaskuläre) Mortalität von Elitesportlern [54]. Die Lebensweise der Elitesportler und deren genetische Voraussetzungen können dabei vorteil- hafter gewesen sein als die Variablen in der allgemeinen Bevölkerung. Dafür haben die Sportler häufiger Rhythmusstörungen $[1,3]$ und Vorhofflimmern $[7,38]$.

Wie intensiv das lebensverlängernde Training sein soll, ist unklar. Ab 45 Jahren ist es auf jeden Fall ratsam, für 30-60 Minuten täglich ein moderates körperliches Training durchzuführen $[4,47]$. Höhere Belastungen (> 41 METs [metabolische Äquivalente]-Stunden/Tag) bringen keinen sicheren Vorteil bezüglich der Überlebensrate und haben eventuell sogar negative Auswirkungen [4]. Farahmand et al. fanden heraus, dass die „langsameren“ Vasa-Lauf-Teilnehmer [18] niedrigere Mortalitätsraten hatten als die „schnellen“ (standardisierte Mortalitätsrate [SMR] $0,48>240 \%$ der Siegerzeit vs. SMR 0,72, 100-120\% der Siegerzeit). Dies spricht für die mäßige Dosierung des ambitionierten Sports.

\section{kurzgefasst}

Die Möglichkeit des PHT in Verbindung mit Sport nimmt im Alter zu. Generell liegt der PHT im Bereich 5/1 Mio./Jahr, bei 55-75-Jährigen 7,5/1 Mio./Jahr. Frauen sind 15-20 mal seltener betroffen.

\section{Screening und Vorsorgeuntersuchungen \\ $\checkmark$}

In der täglichen Routine stellen die ambitionierten Amateursportler und Leistungssportler ca. 50\% der Patienten einer sportärztlich orientierten kardiologischen Praxis dar (eigene Angaben). Die Neuentdeckung des Marathons, des Triathlons und des Sports sind bei den 40-Jährigen nach der ersten Phase des beruflichen Erfolgs häufig Motivation im Sinne der Selbstfindung und Abwechslung.

Bei den ambitionierten Alterssportlern wird das klinische Bild von Hypertonie, Vorhofflimmern [7] und möglicher koronarer Herzerkrankung [61] dominiert. Bei den übrigen 50\% der Amateure besteht die sportmedizinische Arbeit aus Leistungseinschätzung und Indikationsstellung für Bewegungsmaßnahmen aufgrund von koronarer Herzerkrankung, Adipositas, Diabetes mellitus, arterieller Hypertonie, Erhaltung der körperlichen Funktionen im Alter und aufgrund des metabolischen Syndroms. Leistungsoptimierung oder Wettkampfvorbereitung spielen hier eine untergeordnete Rolle. Im Vordergrund stehen vielmehr die Definition der eigenen Leistungsgrenzen/-bereiche und das Aussprechen einer Leistungseinschätzung und Trainingsempfehlung.

\section{Bisherige offizielle Empfehlungen}

Da sportbezogene Todesfälle im Alter und intensive Wettkämpfe (Master-Turniere) zunehmen, stellt sich die Frage, ob die bisherigen offiziellen Empfehlungen zeitgemäß sind [35]. Die Empfehlungen der Gesellschaften, die in den Publikationen von Maron et al. [45] und Corrado et al. [10] wiedergegeben werden, sind zum Teil historisch und ökonomisch geprägt. Die bisherigen offiziellen Konzepte beinhalten die körperliche Untersuchung und die Anamnese des jungen Sportlers [45] - und bei der italienisch geprägten Variante zusätzlich ein Ruhe-EKG [8]. Diese Konzepte mögen für jugendliche Sportler in der Vergangenheit ausgereicht haben, sind aber für die heutigen Verhältnisse nicht hinreichend. Aufgrund der allgemeinen Entwicklung wird auch von überzeugten Vertretern der alleinigen EKG-Diagnostik diese Einstellung 
aufgegeben [66]. Die FIFA hat in ihren Empfehlungen schon eine Vorreiterrolle übernommen und empfiehlt eine sehr fundierte Echokardiographie [19], basierend auf den Publikationen von Dvorak et al. [16] und Thunenkotter et al. [55, 56]. Die Organisation der professionellen Fußballspieler (FifPro) fordert eine Qualitätsverbesserung der medizinischen Diagnostik bezüglich des plötzlichen kardialen Todes [20]. Entsprechend ist eine Belastungsuntersuchung als Ergänzung zur Echokardiographie dringend angeraten. Ein Belastungstest liefert nicht nur Informationen zu einer kardialen Ischämie oder dem Trainingszustand, sondern auch zur arteriellen Hypertonie während der Belastung, zum Herzfrequenzverlauf und zum Auftreten von eventuellen Rhythmusstörungen unter der Belastung. Die Echokardiographie liefert - neben der Beschreibung der kardialen Strukturen (Ausmaß der Hypertrophie, Aorta, Vorhof, Ventrikel-Dimensionen, Myokardstruktur und Wanddicken) $[25,39,65]$ - auch funktionelle Informationen. Die Doppler-Messungen geben Informationen über Blutflussgeschwindigkeiten und diastolische Funktion oder über die Geschwindigkeiten des Myokards (Tissue Doppler Imaging). Die Strain-Technik liefert segmentale und globale myokardiale Werte/Verlaufskurven der Deformation innerhalb des Herzzyklus $[11,38,51]$. Gemessen am zeitlichen und finanziellen Aufwand insgesamt [21] stellen die bisher empfohlenen Maßnahmen ein Minimum dar. In der Literatur werden weitere und immer aufwendigere Untersuchungsverfahren diskutiert, wie z.B. Electron Beam Tomography [6], Angio-CT und MRT [17, 33].

\section{Aktuelle Screening-Vorsorge-Situation im Alltag} Bisherige offizielle Empfehlungen $[10,45]$ unterliegen einem Wandel $[16,17,25,33,35]$. Sie müssten sich an den Empfehlungen für die Patienten mit koronarer Herzerkrankung orientieren [49], aber auch nach den laufenden Erkenntnissen richten $[16,55]$. Nicht nur der PHT stellt eine Komplikation dar, sondern auch strukturelle Veränderungen der Herzens (Aorta/Vorhof/Ventrikel) und die möglichen Herzrhythmusstörungen sind Komplikationsmöglichkeiten. Vorhofflimmern kann einen aktiven, quasi gesunden, Freizeitsportler in einen körperlich wenig aktiven Menschen wandeln. Die gesundheitliche Situation eines ambitionierten Sportlers ist oft sehr komplex und kann bei klinischen Problemen ein breites interdisziplinäres Gebiet zwischen Immunologie, Kardiologie, Orthopädie bis hin zur Zahnmedizin und Kieferorthopädie beanspruchen.

Die folgenden Empfehlungen des Screenings stützen sich auf die zitierte Literatur und auf die 20-jährige Praxis der aktiven Betreuung von Leistungssportlern und Amateuren sowie auf eigene Sporterfahrungen.

Prinzipiell kann man klinisch vier verschiedene Untersuchungsanlässe unterscheiden:

1. Screening-Untersuchung (Eingangsuntersuchung)

2. Allgemeine Vorsorge (laufende Kontrollen im Verlauf)

3. Kontrolluntersuchung bei Beschwerden (Leistungsabfall, Luftnot, Rhythmusstörungen, thorakale Beschwerden, Infekt)

4. Leistungsdiagnostik zwecks Trainingsempfehlung

Aufgrund der möglichen koronaren Herzerkrankung [61], der Belastungshypertonie [40] und der zusätzlichen Informationen durch die Echokardiographie $[25,66]$ können im Bereich des Breiten- und Leistungssports ähnliche Maßnahmen empfohlen werden wie für Sportler mit koronarer Herzerkrankung [49].
Das bedeutet, dass die Echokardiographie und ein Belastungstest als Basis für eine Sporttauglichkeitsuntersuchung für Probanden über 35 Jahre angesehen werden können $[25,61]$.

\section{Screening-Untersuchung (Eingangsuntersuchung)}

Die Eingangsuntersuchung beinhaltet neben der körperlichen Basisuntersuchung und Anamnese folgende Untersuchungen: Echokardiographie, Belastungs-EKG, Lungenfunktionstest und Basisblutuntersuchung (Differenzialblutbild, CRP, Kreatinin, CK, GPT, GOT, GGT, LDH, LDL-/HDL-Cholesterin, Triglyzeride, Blutzucker, Eisen, Ferritin, TSH, Elektrophorese). Als erweitertes Modul erfolgt auch eine Carotis-Duplex-Untersuchung (Bestimmung der Intima-Media-Dicke, Plaques), um den Arteriosklerose-Status zu ermitteln [62]. Bei auffälligem Ruhe- oder Belastungs-EKG erfolgt eine Stress-Echokardiographie [37]. Bei der Echokardiographie werden alle modernen Untersuchungstechniken eingesetzt [36] - einschließlich der Strain-Echokardiographie $[11,38,51]$.

Die Echokardiographie erfasst heute nicht nur das Ausmaß der Hypertrophie, die Größe von Herzkammern und Vorhöfen, die Herzklappenfunktion und die visuelle Beurteilung der Kontraktionen des rechten/linken Ventrikels. Mittels Tissue Doppler Imaging und Strain-Technologie werden komplexe Funktionsmessungen im Bereich beider Kammern und Vorhöfe durchgeführt, die ausführliche objektivierbare Veränderungen vor allem im Verlauf erfassen können ( Abb.4) $[38,51]$. Diese, für den Nicht-Kardiologen hochkomplexen, echokardiographischen Untersuchungen, sind mittlerweile für den erfahrenen (Sport-)Kardiologen Routine geworden.

Grundsätzlich bietet die Spiroergometrie bei Sportlern die Möglichkeit, das physiologische Leistungsprofil zu dokumentieren (u. a. Fettverbrennungsschwelle, aerobe Kapazität, anaerobe Schwelle) und die maximale Sauerstoffaufnahme $\left(\mathrm{VO}_{2} \mathrm{max}\right.$ in $\left.\mathrm{ml} / \mathrm{min} / \mathrm{kg}\right)$ einzuschätzen. Fakultativ wird die Körperzusammensetzung mittels einer komplexeren Impedanz-Waage dokumentiert. Bei Breitensportlern und Patienten mit metabolischem Syndrom wird die Spiroergometrie angewendet, um den Leistungszustand zu überprüfen und eine aerobe Trainingsgestaltung zu planen [59]. Hier liegt der Schwerpunkt auf der Fettverbrennung und schonenden Aktivitätsplanung im aeroben Bereich [30].

Screening mit Strahlendiagnostik (Kardio-CT, Electron Beam Tomography, Angio-CT) [17] hat sich bisher in der Routine aus Kostengründen und fehlender Akzeptanz (Strahlenbelastung) bei vorhandenen Alternativen (Carotis-Duplex/Stress-Echokardiographie/Strain/MRT) nicht durchgesetzt. Sie spielt aber in der Literatur $[6,17]$ und möglicherweise in der Zukunft eine wichtigere Rolle [14].

Sportmedizinische Belastungsuntersuchungen in Deutschland werden mittlerweile bis zu 80\% auch durch die gesetzlichen Krankenkassen bezuschusst [32]. Bei einer bestimmten Fragestellung (Myokarditis/Fibrose) oder bei Verdacht auf arrhythmogene rechtsventrikuläre Kardiomyopathie (extrem selten) wird eine Cardio-MRT Untersuchung eingesetzt [5]. 


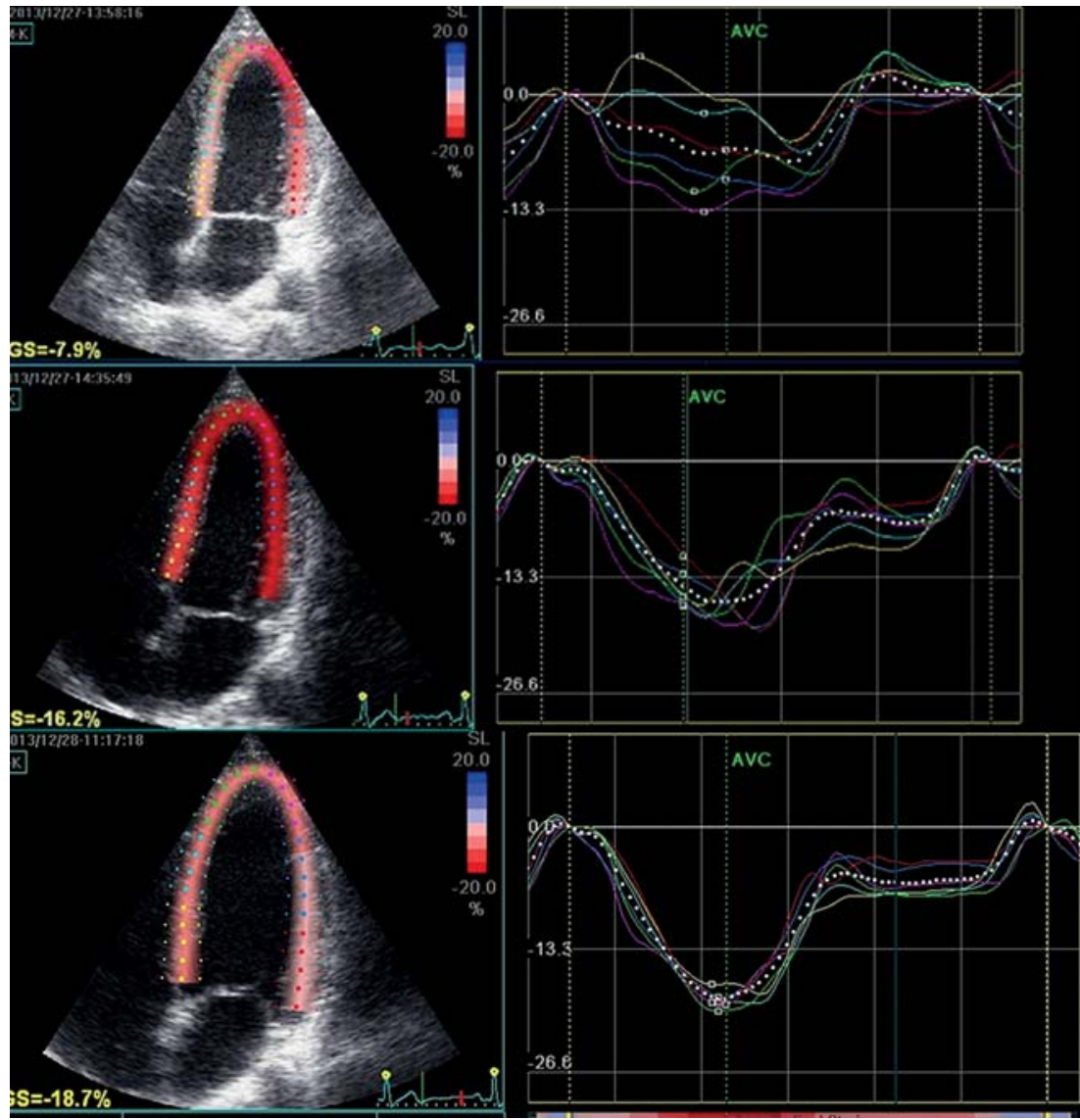

Abb.4 Langsame Besserung der Deformation des linken Ventrikels (LV) nach Konversion in den Sinusrhythmus. Oben: Deformation der LV-Segmente während des Vorhofflimmerns. Mitte: 5 Minuten nach Konversion in den Sinusrhythmus. Unten: 24 Stunden danach Normalisierung der segmentalen Deformation nach der Kardioversion.

\section{Allgemeine Kontrollen}

\section{$\nabla$}

Bei den meist jährlich durchgeführten Kontrollen erfolgen immer eine Echokardiographie und ein Belastungstest. Besonderen Wert legen wir auf die Dokumentation der Belastungshypertonie [40]. Eine Echokardiographie erfolgt hauptsächlich, um die Größe der Kammern/Vorhöfe zu vergleichen und eventuelle Veränderungen im Bereich der Herzklappen und der Aorta zu erfassen [65]. Eine Aortenerweiterung [65] mit konsekutiver Aortenklappeninsuffizienz ist bei Ausdauersportlern nicht selten (2\%) [39]. Bei ambitionierten und Wettkampfsportlern erfolgt außerhalb der WettkampfSaison oft eine Spiroergometrie, um die Trainingsplanung zu überprüfen; bei Leistungssportlern auch in der Vor-Wettkampfphase.

\section{Kontrolluntersuchung bei Beschwerden}

Hier richtet sich die Untersuchung nach der klinischen Situation. Alle neuen Ruhe-EKG-Veränderungen, Synkopen und Luftnot müssen konsequent differenzialdiagnostisch abgeklärt werden. Neben der Basisuntersuchung mittels Echokardiographie (inkl. Doppler, Tissue Doppler Imaging, Strain-Technologie) und Ruhe-EKG erfolgen alle weiteren Untersuchungen nach klinischem Bedarf (Stress-Echokardiographie, Langzeit-EKG, Angio-CT, Cardio-MRT bis zur Koronarangiographie oder elektrophysiologischen Untersuchung).

Eine ausführliche Blutuntersuchung, inklusive der Überprüfung der Virus-Antikörper (Ebstein-Barr-, Zytomegalie-, Herpes-, Parvo-, Coxsackie-, Echo-Viren etc.) und der bakteriellen Antikörper (Chlamydia pneumoniae [58], Borrelien) sollte im Falle eines Leistungsabfalls vorgenommen werden. Negative Befunde der Blutuntersuchung bedeuten keinen Ausschluss einer Myokarditis. Mit diesem Vorgehen haben wir bis jetzt keinen Todesfall oder eine unerkannte Myokarditis im Verlauf erfahren.

\section{Leistungsdiagnostik}

Die Diagnostik mittels Spiroergometrie ist extrem wichtig bei der Trainingsplanung und der Leistungsbeurteilung bei aktiven Sportlern [39] sowie bei der Aktivitätsplanung der betroffenen Patienten [30]. Nur die Spiroergometrie liefert in der Gesamtheit der Daten eine optimale Beurteilung des Sportlers/des Patienten bezüglich der Fettverbrennung, der Trainingsbereiche und der Leistungseinschätzung.

\section{kurzgefasst}

Screening der Amateure und der ambitionierten Sportler beinhaltet 4 Möglichkeiten: Eingangsuntersuchung, allgemeine Vorsorge, Kontrolluntersuchung bei Beschwerden und reine Leistungsdiagnostik zwecks Trainingssteuerung. Neben der klinischen Untersuchung und der Anamnese sind Echokardiographie und Belastungstest die Basisuntersuchungen erweitert durch die Blutuntersuchungen, Langzeit-EKG und CardioMRT. Die Spiroergometrie ermöglicht eine umfangreiche Einschätzung des Leistungsprofils des Sportlers/Patienten.

\section{Schlussbemerkung}

$\nabla$

Die Kosten-Nutzen-Relation einer kombinierten BelastungsEKG-/Echokardiographie-Untersuchung oder Spiroergometrie ist gemessen am Gesamtaufwand der ambitionierten sportlichen Betätigung akzeptabel. Gerade durch die Beteiligung der gesetzlichen Krankenkassen an den Kosten [32] ist die medizinische Versorgung der Sportler zumindest in Deutschland gewährleistet. Aber auch in anderen Ländern müssten die etwaigen Screening-Kosten in Anbetracht der aufwendigen Kosten 
der Vorbereitung und Teilnahme an Marathon- oder TriathlonWettbewerben eher zu vernachlässigen sein [21].

Sport hat eine große soziale und mediale Bedeutung. Entsprechend hat die Prävention der plötzlichen sportbezogenen Todesfälle oder eines „negativen kardialen Remodellings durch Sport“ nicht nur eine individuelle Komponente, sondern auch eine weitreichende soziale Bedeutung für die körperliche Aktivität der allgemeinen Bevölkerung [57]. Deswegen sind auch hier weitere Aufwendungen, Studien und Screenings der Sportler in der industrialisierten Welt wichtig und finanziell vertretbar.

\section{Konsequenz für Klinik und Praxis}

- Die sportlichen Aktivitäten in der allgemeinen Bevölkerung bis zu anstrengenden Wettkämpfen im Bereich des Triathlons und Marathons haben deutlich zugenommen.

- Vor diesem Hintergrund und in Anbetracht der lang andauernden Karriere der professionellen Sportler bedarf es klarer Empfehlungen zu Vorsorgeuntersuchungen, um den plötzlichen Herztod in Verbindung mit Sport zu vermeiden und auch „negatives kardiales Remodelling“ durch exzessive sportliche Betätigung zu verhindern. Gerade beim Leistungsabfall des Sportlers ist eine genaue Untersuchung notwendig. - Auch bei der Planung der sportlichen Aktivität oder des Trainings der Freizeitsportler/Patienten, ist eine Vorsorgeuntersuchung/Leistungsdiagnostik mit Spiroergometrie sinnvoll.

Autorenerklärung: Die Autoren erklären, dass sie keine finanzielle Verbindung mit einer Firma haben, deren Produkt in diesem Beitrag eine Rolle spielt (oder mit einer Firma, die ein Konkurrenzprodukt vertreibt).

Literatur

1 Andersen K, Farahmand B, Ahlbom A et al. Risk of arrhythmias in 52755 long-distance cross-country skiers: a cohort study. Eur Heart J 2013; 34: 3624-3631

2 Augsburger Allgemeine. Freizeit-Sportler stirbt beim Triathlon in Roth (10.07.2011). http://www.augsburger-allgemeine.de/newsticker/Freizeit-Sportler-stirbt-beim-Triathlon-in-Roth-id15844541.html (letzter Zugriff: 10.09.2014)

3 Baldesberger S, Bauersfeld $U$, Candinas $R$ et al. Sinus node disease and arrhythmias in the long-term follow-up of former professional cyclists. Eur Heart J 2008; 29: 71-78

4 Bellavia A, Bottai M, Wolk A et al. Physical activity and mortality in a prospective cohort of middle-aged and elderly men - a time perspective. Int J Behav Nutr Phys Act 2013; 10: 94

5 Breuckmann F, Mohlenkamp S, Nassenstein K et al. Myocardial late gadolinium enhancement: prevalence, pattern, and prognostic relevance in marathon runners. Radiology 2009; 251: 50-57

6 Budoff MJ, Shokooh S, Shavelle RM et al. Electron beam tomography and angiography: sex differences. Am Heart J 2002; 143: 877-882

7 Calvo N, Brugada J, Sitges $M$ et al. Atrial fibrillation and atrial flutter in athletes. Br J Sports Med 2012; 46 (Suppl. 01): i37-43

8 Corrado D, Basso C, Rizzoli G et al. Does sports activity enhance the risk of sudden death in adolescents and young adults?. J Am Coll Cardiol 2003; 42: 1959-1963

9 Corrado D, Pelliccia A, Heidbuchel $H$ et al. Recommendations for interpretation of 12-lead electrocardiogram in the athlete. Eur Heart J 2010; 31: 243-259

10 Corrado D, Schmied C, Basso C et al. Risk of sports: do we need a pre-participation screening for competitive and leisure athletes?. Eur Heart J 2011; 32: 934-944
11 D'Andrea A, Cocchia R, Riegler $L$ et al. Left ventricular myocardial velocities and deformation indexes in top-level athletes. J Am Soc Echocardiogr 2010; 23: 1281-1288

12 Danaei G, Ding EL, Mozaffarian D et al. The preventable causes of death in the United States: comparative risk assessment of dietary, lifestyle, and metabolic risk factors. PLoS Med 2009; 6: e1000058

13 Dickhuth H, Hipp A, Niess A et al. Differenzialdiagnostik der physiologischen Herzhypertrophie (Sportherz). Dtsch Z Sportmed 2001; 52: 205210

14 Doh JH, Koo BK, Nam CW et al. Diagnostic value of coronary CT angiography in comparison with invasive coronary angiography and intravascular ultrasound in patients with intermediate coronary artery stenosis: results from the prospective multicentre FIGURE-OUT (Functional Imaging criteria for GUiding REview of invasive coronary angiOgraphy, intravascular Ultrasound, and coronary computed Tomographic angiography) study. Eur Heart J Cardiovasc Imaging 2014; 15: 870-877

15 Drezner JA, Ackerman MJ, Anderson J et al. Electrocardiographic interpretation in athletes: the 'Seattle criteria'. Br J Sports Med 2013; 47: 122-124

16 Dvorak J, Grimm K, Schmied C et al. Development and implementation of a standardized precompetition medical assessment of international elite football players - 2006 FIFA World Cup Germany. Clin J Sport Med 2009; 19: 316-321

17 Erbel R, Budoff $M$. Improvement of cardiovascular risk prediction using coronary imaging: subclinical atherosclerosis: the memory of lifetime risk factor exposure. Eur Heart J 2012; 33: 1201-1213

18 Farahmand BY, Ahlbom A, Ekblom $O$ et al. Mortality amongst participants in Vasaloppet: a classical long-distance ski race in Sweden. J Intern Med 2003; 253: 276-283

19 FIFA. Pre-Competition Medical Assessment (PCMA). 2009; http://www.fifa.com/mm/document/afdeveloping/medical/01/07/26/86/fifapcmaform.pdf (letzter Zugriff: 10.09.2014)

20 FifPro. Status of Pre-Competition Medical Assessment (20.03.2014). http://www.fifpro.org/en/news/the-quality-of-pre-competition-medicalassessment (letzter Zugriff: 10.09.2014)

21 FoxBusiness. Gabrielle Karol: The True Costs of Training for Triathlons (16.07.2013). http://www.foxbusiness.com/personal-finance/2013/07/16/true-costs-training-for-triathlons) (letzter Zugriff: 10.09.2014)

22 Frost F. The dubious origin of marathon. Am J ancHist 1979; 4: 159-163

23 Gati S, Sheikh N, Ghani S et al. Should axis deviation or atrial enlargement be categorised as abnormal in young athletes? The athlete's electrocardiogram: time for re-appraisal of markers of pathology. Eur Heart J 2013; 34: 3641-3648

24 George KP, Wolfe LA, Burggraf GW. The 'athletic heart syndrome'. A critical review. Sports Med 1991; 11: 300-330

25 Grazioli G, Merino B, Montserrat S et al. Usefulness of Echocardiography in Preparticipation Screening of Competitive Athletes. Rev Esp Cardiol 2014; 67: 701-705

26 Hamel P, Simoneau JA, Lortie G et al. Heredity and muscle adaptation to endurance training. Med Sci Sports Exerc 1986; 18: 690-696

27 Harris KM, Henry JT, Rohman E et al. Sudden death during the triathlon. JAMA 2010; 303: 1255-1257

28 Hart G. Exercise-induced cardiac hypertrophy: a substrate for sudden death in athletes? Exp Physiol 2003; 88: 639-644

29 Henschen S. Skilanglauf und Skiwettlauf. Eine medizinische Sportstudie. Mitt Med Klin Upsala 1899; 2: 74

30 Hewitt JA, Whyte GP, Moreton $M$ et al. The effects of a graduated aerobic exercise programme on cardiovascular disease risk factors in the NHS workplace: a randomised controlled trial. J Occup Med Toxicol 2008; 3: 7

$31 \mathrm{Kim} J \mathrm{H}$, Malhotra R, Chiampas G et al. Cardiac arrest during long-distance running races. N Engl J Med 2012; 366: 130-140

32 Krankenkasse: Techniker Krankenkasse. Sportmedizinische Untersuchung (19.07.2012). 2013; http://www.tk.de/tk/behandlungsangebote/besondere-tk-angebote/sportmedizinische-untersuchung/460540 (letzter Zugriff: 10.09.2014)

33 La Gerche A, Baggish AL, Knuuti J et al. Cardiac imaging and stress testing asymptomatic athletes to identify those at risk of sudden cardiac death. JACC Cardiovasc Imaging 2013; 6: 993-1007

34 Leischik R. Endurance Sport and Cardiac Injury. Polish Journal of Cardiology, Kardiologia Polska 2014; 72: 311-321 
35 Leischik R. Ugly duckling or Nosferatu? - marathon running and cardiovascular function How to screen the athletes?. Eur Heart J 2014; published: 13.02.2014. http://eurheartj.oxfordjournals.org/content/early/2014/01/08/eurheartj.eht502/reply\#ehj_el_2040 (letzter Zugriff: 10.09.2014)

36 Leischik R, Bartel T, Möhlenkamp S et al. Stress echocardiography: new techniques. Eur Heart J 1997; 18: 49-56

37 Leischik R, Dworrak B, Littwitz H et al. Prognostic significance of exercise stress echocardiography in 3329 outpatients (5-year longitudinal study). Int J Cardiol 2007; 119: 297-305

38 Leischik R, Littwitz H. Slow recovery of the right and left ventricular deformation after conversion from atrial fibrillation. American Journal of Sports Science 2014; 2 : 13-16

39 Leischik R, Spelsberg N. Endurance Sport and "Cardiac Injury": A Prospective Study of Recreational Ironman Athletes. Int J Environ Res Public Health 2014; 11: 9082-9100

40 Leischik R, Spelsberg N, Niggemann $H$ et al. Exercise-induced arterial hypertension - an independent factor for hypertrophy and a ticking clock for cardiac fatigue or atrial fibrillation in athletes?. F1000Research 2014; 3: 105 http://f1000r.es/3b4 (letzter Zugriff: 10.09.2014)

41 Marijon E, Bougouin W, Perier MC et al. Incidence of sports-related sudden death in France by specific sports and sex. JAMA 2013; 310: 642-643

42 Marijon E, Tafflet M, Antero-Jacquemin J et al. Mortality of French participants in the Tour de France (1947-2012). Eur Heart J 2013; 34: 31453150

43 Marijon E, Tafflet M, Celermajer DS et al. Sports-related sudden death in the general population. Circulation 2011; 124: 672-681

44 Maron BJ, Pelliccia A. The heart of trained athletes: cardiac remodeling and the risks of sports, including sudden death. Circulation 2006; 114: 1633-1644

45 Maron BJ, Thompson PD, Ackerman MJ et al. Recommendations and Considerations Related to Preparticipation Screening for Cardiovascular Abnormalities in Competitive Athletes: 2007 Update A Scientific Statement From the American Heart Association Council on Nutrition, Physical Activity, and Metabolism: Endorsed by the American College of Cardiology Foundation. Circulation 2007; 115: 1643-1655

46 Marti B, Goerre S, Spuhler T et al. Plötzliche Todesfälle an Schweizer Volksläufen 1978-1987: eine epidemiologisch-pathologische Studie. Schweiz Med Wochenschr 1989; 119: 473-482

47 O'Keefe JH, Harshal RP, Lavie CJ et al. Potential Adverse Cardiovascular Effects From Excessive Endurance Exercise. Mayo Clin Proc 2012; 87: 587595

48 Pelliccia A. Filling the gap of understanding the athlete's ECG. Eur J Prev Cardiol 2013; 20: 9-11

49 Pelliccia A, Fagard R, Bjørnstad HH et al. Recommendations for competitive sports participation in athletes with cardiovascular disease A consensus document from the Study Group of Sports Cardiology of the Working Group of Cardiac Rehabilitation and Exercise Physiology and the Working Group of Myocardial and Pericardial Diseases of the European Society of Cardiology. Eur Heart J 2005; 26: 1422-1445

50 Priori SG, Aliot E, Blomstrom-Lundqvist C et al. Task Force on Sudden Cardiac Death, European Society of Cardiology. Europace 2002; 4: 3-18

51 Sengupta PP, Krishnamoorthy VK, Korinek J et al. Left ventricular form and function revisited: applied translational science to cardiovascular ultrasound imaging. J Am Soc Echocardiogr 2007; 20: 539-551

52 Sheikh N, Papadakis M, Ghani S et al. Comparison of electrocardiographic criteria for the detection of cardiac abnormalities in elite black and white athletes. Circulation 2014; 129: 1637-1649

53 Stewart KJ. Exercise training and the cardiovascular consequences of type 2 diabetes and hypertension: plausible mechanisms for improving cardiovascular health. JAMA 2002; 288: 1622-1631

54 Teramoto M, Bungum TJ. Mortality and longevity of elite athletes. J Sci Med Sport 2010; 13: 410-416

55 Thunenkotter T, Schmied C, Dvorak J et al. Benefits and limitations of cardiovascular pre-competition screening in international football. Clin Res Cardiol 2010; 99: 29-35

56 Thunenkotter T, Schmied C, Grimm K et al. Precompetition cardiac assessment of football players participating in the 2006 FIFA World Cup Germany. Clin J Sport Med 2009; 19: 322-325
57 Warburton DE, Nicol CW, Bredin SS. Health benefits of physical activity: the evidence. CMAJ 2006; 174: 801-809

58 Wesslen L, Pahlson C, Lindquist 0 et al. An increase in sudden unexpected cardiac deaths among young Swedish orienteers during 1979-1992. Eur Heart J 1996; 17: 902-910

59 Whelton SP, Chin A, Xin X et al. Effect of aerobic exercise on blood pressure: a meta-analysis of randomized, controlled trials. Ann Intern Med 2002; 136: 493-503

60 Whyte G, Sheppard M, George K et al. Post-mortem evidence of idiopathic left ventricular hypertrophy and idiopathic interstitial myocardial fibrosis: is exercise the cause?. BMJ Case Rep 2008; 42: 304-306

61 Wike J, Kernan M. Sudden cardiac death in the active adult: causes, screening, and preventive strategies. Curr Sports Med Rep 2005; 4: 76-82

62 Willeit J, Kiechl S. Prevalence and risk factors of asymptomatic extracranial carotid artery atherosclerosis. A population-based study. Arterioscler Thromb 1993; 13: 661-668

63 Wilson M, O'Hanlon R, Prasad S et al. Diverse patterns of myocardial fibrosis in lifelong, veteran endurance athletes. J Appl Physiol (1985) 2011; 110: $1622-1626$

64 World Health Organisation. Global Health Risks. Mortality and burden of disease attributable to selected major risks. 2009; http://www.who.int/healthinfo/global_burden_disease/GlobalHealthRisks_report_full.pdf (letzter Zugriff: 10.09.2014)

65 Yim ES. Aortic root disease in athletes: aortic root dilation, anomalous coronary artery, bicuspid aortic valve, and Marfan's syndrome. Sports Med 2013; 43: 721-732

66 Yim ES, Basilico F, Corrado G. Early screening for cardiovascular abnormalities with preparticipation echocardiography: utility of focused physician-operated echocardiography in preparticipation screening of athletes. J Ultrasound Med 2014; 33: 307-313

67 Maron BJ, Doerer IJ, Haas TS et al. Sudden deaths in young competitive athletes analysis of 1866 Deaths in the United States, 1980-2006. Circulation 2009; 119: 1085-1092

68 Solberg EE, Gjertsen F, Haugstad E, Kolsrud L. Sudden death in sports among young adults in Norway. Eur J Cardiovasc Prev Rehabil 2010; 17: 337-341 